\title{
Atenção e Comportamento Inibitório em Crianças de 6 a 8 Anos
}

\author{
Paula Lemes ${ }^{1}$ \\ Joaquim Carlos Rossini \\ Universidade Federal de Uberlândia
}

\begin{abstract}
RESUMO - Muitas evidências sugerem que os processos atentivos apresentam diferenças ao longo do desenvolvimento. O presente estudo avaliou a capacidade atentiva e o controle inibitório de 130 crianças de seis a oito anos de idade em uma tarefa comportamental agir/não agir adaptada para esta faixa etária. Os resultados sugerem que as crianças de seis anos apresentaram tempos de resposta significativamente superiores e cometeram mais erros quando comparadas às crianças de oito anos. Esses achados corroboram estudos que apontam diferenças importantes no processamento da informação nessa faixa etária, principalmente no que se refere à capacidade de controle atentivo, relacionada a comportamentos desatentos frente a um objetivo, e à capacidade de controle inibitório, relacionada a comportamentos impulsivos de resposta motora.
\end{abstract}

Palavras-chave: atenção, resposta inibitória, desenvolvimento cognitivo, tarefa agir/não agir

\section{Attention and Inhibitory Behavior in Children of 6 to 8 Year-olds}

\begin{abstract}
Considerable evidence suggests that attention and inhibitory behavior show differences along specific developmental stages. The present study aimed to evaluate the attention and inhibitory control of 130 children, aged six to eight years, in a go/no-go task adapted for this age group. The results suggest that 6-year-old children had significantly higher reaction times and made more errors when compared to 8-year-old children. These findings corroborate studies that indicate important differences in information processing in the age group studied, especially with regard to the attentional control capacity, related to inattentive behaviors when a goal is proposed, and the inhibitory control capacity, related to impulse response behaviors.
\end{abstract}

Keywords: attention, inhibitory response, cognitive development, go/no-go task

Frequentemente somos obrigados a interromper determinado comportamento para iniciar outro mais adequado. Essa habilidade de inibir uma ação para iniciar outra mais condizente às demandas internas e externas é fundamental para o processo de adaptação comportamental e envolve vários aspectos dos processos atentivos. Dentre esses, o processo inibitório pode ser considerado como a supressão de pensamentos, ações e emoções, ou como processos de modificação comportamental que representam um mecanismo fundamental no sistema de controle da ação (Logan, 1994; Verbruggen \& Logan, 2008). Assim, a capacidade inibitória é regulada por múltiplos componentes de uma ampla gama de processos que, por sua vez, integram as funções executivas (Barkley, 1997). Nesse sentido, um número considerável de estudos têm apontado mudanças cognitivas significativas na faixa etária compreendida entre cinco e oito anos de idade (Bartgis, Lilly \& Thomas, 2003; Bartgis, Thomas, Lefler \& Hartung, 2008; Davis, Bruce, Snyder \& Nelson, 2003; Ridderinkhof \& van der Molen, 1995).

De modo geral, essas investigações apontam que crianças mais novas (cinco a seis anos) apresentam maior dificuldade em selecionar a informação relevante do ambiente e uma capacidade limitada de ignorar estímulos irrelevantes,

1 Endereço para correspondência: Programa de Pós-Graduação em Psicologia, Universidade Federal de Uberlândia, UFU, Av. Pará, 1720, Bloco 2C Sala 29 , Campus Umuarama, Uberlândia, MG, Brasil, CEP:38400-902.E-mail: paulinha_lemes@hotmail.com quando comparadas a crianças mais velhas (10 a 12 anos) (Bartgis et al., 2003; Ridderinkhof \& van der Molen, 1995). Tal fato sugere um rápido desenvolvimento da capacidade cognitiva nessa faixa etária (Korkman, Kemp \& Kirk, 2001) e o desenvolvimento de ações integradas de vários fatores cognitivos, entre esses, o aumento na capacidade inferencial e uma melhoria significativa dos recursos mnemônicos (Roberts \& Pennington, 1996). Nigg (2000) argumenta que a relação entre diferentes tipos de inibição é importante para o melhor entendimento de vários processos de seleção em diferentes etapas do desenvolvimento, bem como em diferentes condições clínicas. Para tanto, esse autor propõe uma distinção dos componentes cognitivos que integram a capacidade inibitória, distinguindo a inibição em duas classes gerais de ações: a inibição da ação (com característica exógena e executiva) e a inibição atentiva (com característica endógena e automática). Essas duas classes tipológicas compreendem três tipos de inibição. Primeiramente, a inibição da ação propriamente dita, com impacto na adaptação comportamental imediata (e.g., tarefas de desempenho contínuo de reforço imediato; Newman, Widom \& Nathan, 1985). O segundo tipo de inibição seria responsável pela supressão de comportamentos, visando uma melhora adaptativa a médio e longo prazo (e.g., tarefas de tolerância ao atraso do reforço; Logan \& Cowan, 1984) e, por fim, processos inibitórios definidos por fatores endógenos e automáticos como a habituação e a inibição de retorno nas tarefas visuais (Klein, 2000). Esses componentes regulam ações atentivas autodirigidas e autorreguladoras relacionadas 
às adaptações comportamentais em ambientes dinâmicos que envolvem, em maior ou em menor grau, ações inibitórias tanto na preparação da ação motora, quanto na interrupção de um padrão comportamental predominantemente estabelecido (Barkley, 2008; Verbruggen, \& Logan, 2008).

Por meio de uma abordagem investigativa que utilizou métodos neurofisiológicos (Potencial Relacionado a Eventos: ERP- P3) associados a medidas comportamentais na investigação da capacidade atentiva em crianças e adultos, Ridderinkhof e van der Molen (1995) obtiveram resultados que sugerem que crianças entre 5 e 6 anos de idade são naturalmente mais dispersas, apresentam respostas motoras lentas e cometem, de modo geral, um maior número de erros quando comparadas às crianças mais velhas.

Sob o ponto de vista comportamental, Hamdan e Pereira (2009) apontam que uma das tarefas mais utilizadas em avaliações das funções executivas é a do tipo agir/não agir (go/no-go task). Nessa tarefa, uma série de estímulos, visuais ou sonoros, é apresentada aos participantes, que são instruídos previamente a responder, o mais rapidamente possível, à presença de um estímulo agir específico, e a suprimir a resposta na presença de um estímulo não agir (e.g., pressionar uma determinada tecla com a apresentação da letra K, mas não efetuar nenhuma resposta com a apresentação da letra L). Esse tipo de tarefa provê três índices importantes acerca do comportamento: a probabilidade de executar a resposta em uma prova não agir (erro de coomissão); a probabilidade da ausência de resposta em uma prova agir (erro de omissão) e o tempo de reação (TR) nas provas agir (velocidade do processamento) (Riccio, Reynolds \& Lowe, 2001).

Rubia, Taylor, et al. (2001) realizaram uma avaliação neuropsicológica em 55 indivíduos de 7 a 15 anos de idade, distribuídos em três grupos: indivíduos com diagnóstico de Transtorno de Déficit de Atenção e Hiperatividade (TDAH) $(\mathrm{n}=16)$, indivíduos sem diagnóstico de transtornos psiquiátricos (grupo controle) $(\mathrm{n}=23)$ e indivíduos com diagnóstico de outros transtornos psiquiátricos diferentes do TDAH $(\mathrm{n}=16)$. A bateria de testes neuropsicológicos utilizada, denominada Bateria de Tarefas MARS (MARS Task Battery), era composta pelas seguintes tarefas: tarefa agir/não agir (go/no-go task), tarefa de parada (stop task), tarefa de reversão (reversal task), uma tarefa motora do tipo teste da batida do dedo (inhibition of synchronised fingertapping), e uma tarefa de estimativa temporal (delay task). Esse trabalho também investigou, por meio do registro de imagens de ressonância magnética funcional, o padrão de ativação neuronal de adolescentes com o diagnóstico de TDAH $(n=7)$ e de adolescentes sem diagnóstico de transtornos psiquiátricos $(n=9)$, em três tarefas das cinco que compõem a Bateria MARS, sendo essas a tarefa de parada, a tarefa de estimativa temporal e o teste da batida do dedo. Frente à hipótese de que indivíduos com TDAH podem apresentar déficits na alternância entre os processos executórios e inibitórios, os autores encontraram evidências que sugerem que o grupo composto por indivíduos com diagnóstico de TDAH apresenta maior prejuízo na inibição motora quando comparado ao grupo controle; não foram encontradas diferenças significativas no controle inibitório observado no grupo de indivíduos com outros transtornos psiquiátricos e o grupo com TDAH. Em relação ao tempo de processamento das respostas motoras, nenhum grupo de participantes apresentou qualquer prejuízo, porém os participantes com TDAH apresentaram maior variabilidade nas respostas, um número maior de erros de omissões e uma menor probabilidade de inibição motora na tarefa agir/ não agir (dados comportamentais). Em relação às áreas cerebrais envolvidas nas tarefas, há uma subativação do lobo pré-frontal direito no grupo com TDAH, associado a uma ativação reduzida das áreas frontoestriatais. Os investigadores apontam a necessidade de estudos na área com maior número de participantes e um maior controle dos outros transtornos diferentes do TDAH.

Com o objetivo de ampliar os achados de Rubia, Taylor, et al. (2001) sob o aspecto do desenvolvimento, Rubia, Russell, et al. (2001) realizaram o registro de imagens cerebrais por ressonância magnética funcional de adultos saudáveis com idades entre 26 e 58 anos, durante a realização de duas tarefas do tipo agir/não agir e três tarefas do tipo sinal de parada. Esse estudo revelou que, na idade adulta, os mecanismos cerebrais envolvidos na realização das tarefas investigadas apresentam áreas cerebrais comuns na realização das duas tarefas agir/ não agir, áreas comuns na execução das três tarefas de sinal de parada e áreas comuns na realização das cinco tarefas em conjunto. Em relação às áreas que envolveram as cinco tarefas, os padrões de ativação neuronal envolveram os córtices medial, frontal inferior e parietal. Em relação à especialização cerebral na execução da tarefa agir/não agir, houve uma maior ativação da região frontoparietal esquerda para respostas seletivas envolvidas na tarefa.

Em outro trabalho que alia dados comportamentais e neurofisiológicos (ERP-P3) de crianças de seis anos de idade e adultos, Davis et al. (2003) avaliaram a tarefa agir/não agir na qual os participantes deveriam responder o mais rápido possível às letras apresentadas na tela de um computador (estímulos agir), excetuando a resposta quando a letra $\mathrm{X}$ fosse apresentada (estímulo não agir). Os resultados dessa investigação corroboram a interpretação de Ridderinkhof e van der Molen (1995) acerca de um processo de maturação acentuado na faixa etária avaliada e apontam latências P3 distintas entre crianças de 6 anos e adultos. Os adultos apresentaram maiores latências quando o estímulo não agir era apresentado, uma vez que este, provavelmente, eliciava um processo de tomada de decisão. Por outro lado, as crianças de seis anos apresentaram latências semelhantes tanto na presença de estímulos agir quanto na presença de estímulos não agir. Os autores interpretaram esses resultados sob dois aspectos: há a possibilidade dos adultos terem utilizado mais recursos cognitivos nas condições de inibição comportamental em comparação às crianças que teriam utilizado recursos de mesma natureza para ambos os estímulos. Uma segunda interpretação seria que os recursos atentivos e a capacidade inibitória não estariam suficientemente desenvolvidos nas crianças de 6 anos.

Outro estudo similar, realizado por Johnstone, Pleffer, Barry, Clarke e Smith (2005), investigou os índices comportamentais e eletrofisiológicos associados à resposta inibitória em crianças, adultos jovens e adultos. Os resultados indicam que as respostas a estímulos de ocorrência predominante (agir) são significativamente mais lentas 
quando efetuadas por crianças do que quando efetuadas por adultos. Resultados comparáveis foram encontrados nas análises dos erros, especificamente nos erros de coomissão nas respostas aos estímulos não agir, mostrando porcentagens maiores desse tipo de erro com as crianças, bem como a progressiva melhora no desempenho dos participantes com o aumento da idade. Em relação à acurácia das respostas, as crianças apresentaram menos acertos quando comparadas aos adultos, o que sugere um aumento de acertos ao longo das faixas etárias investigadas.

Nesse sentido, o desenvolvimento neurocognitivo apresenta mudanças importantes ao longo da vida no que se refere às funções atentivas e ao controle inibitório da ação (para uma revisão sobre controle inibitório na adolescência, ver Jaeger, 2013). Porém, ainda é escasso o número de trabalhos na literatura que investigam quais áreas cerebrais estão envolvidas no desenvolvimento e na maturação destes processos cognitivos em várias fases do desenvolvimento. Em um estudo conduzido por Rubia et al. (2006), a ativação de áreas cerebrais relacionadas à realização de três tarefas comportamentais (tarefa agir/não agir, tarefa de Simon e tarefa de alternância) foi investigado em dois grupos etários (adolescentes e adultos) por meio de ressonância magnética funcional. $O$ resultado dessa pesquisa sugere uma maturação progressiva dos processos de controle cognitivo ao longo do desenvolvimento compreendido entre a infância e meados da idade adulta. Os dados sugerem também que áreas frontoestriatais e frontocorticais estão envolvidas no controle cognitivo e que essa função cognitiva mobiliza um número crescente de áreas cerebrais ao longo do desenvolvimento.

Em um trabalho mais recente, Cragg e Nation (2008) utilizaram uma versão modificada da tarefa agir/não agir para investigar os processos em que a resposta de inibição torna-se mais eficiente durante a infância. Para isso, os autores propuseram algumas condições experimentais em que estímulos agir/não agir foram apresentados de maneira não verbal a 90 crianças distribuídas em dois grupos etários ( 5 a 7 anos e 9 a 11 anos de idade). O resultado desse estudo sugere que as crianças mais novas apresentam mais dificuldade em inibir a resposta ao estímulo não agir quando comparadas às crianças mais velhas. Em outro estudo, Bezdjian, Baker, Lozano e Raine (2009) observaram que o desempenho de 1151 crianças de 9 a 10 anos de idade em uma tarefa agir/não agir e os escores observados em questionários que avaliam comportamentos relacionados à desatenção e à hiperatividade/ impulsividade, respondidos pelos responsáveis das crianças participantes, apresentam uma correlação significativa. Ou seja, os erros de omissões na tarefa agir/não agir apresentam uma correlação positiva significativa com os comportamentos de desatenção, enquanto que os erros de coomissões se correlacionam de maneira positiva e significativa com os comportamentos associados à hiperatividade/impulsividade.

Em âmbito nacional, Miranda, Sinnes, Pompeia e Bueno (2009) descreveram o desempenho de crianças de quatro e cinco anos de idade no teste norte-americano Conners' Kiddie CPT9 (K-CPT) (Conners, 2002). Esse teste utiliza figuras, ao invés de letras, como estímulos imperativos de resposta ou não resposta. Para verificar o desempenho da amostra brasileira, Miranda et al. (2009) analisaram algumas variáveis, dentre elas os tempos de reação (TRs) e a taxa de erros (omissão ou coomissão). Os resultados confirmaram diferenças significativas no desenvolvimento do processo inibitório com TRs menores e menores taxas de erros ao longo das faixas etárias investigadas.

No intuito de avançar na investigação dos processos maturacionais do controle atentivo e inibitório, a presente investigação avaliou o desempenho de crianças de seis a oito anos de idade em uma tarefa agir/não agir, fazendo uso de estímulos similares aos propostos por Conners (2002) e utilizados por Miranda et al. (2009). Na presente pesquisa, aspectos relacionados aos comportamentos de desatenção e impulsividade foram analisados em uma amostra de crianças sem demanda clínica.

\section{Método}

\section{Participantes}

Trezentas crianças, selecionada por conveniência, sem queixas clínicas, com visão normal ou corrigida, com idade entre seis a oito anos e estudantes de uma escola da rede pública de ensino, foram convidadas a participar da presente pesquisa. Apenas as crianças devidamente autorizadas pelo(s) seu(s) responsável (is) legal (is) participaram da pesquisa, resultando em uma amostra de 161 crianças. Dessa amostra, 31 crianças foram excluídas do processo de análise devido a problemas no registro das respostas. A amostra final foi composta por 50 crianças com seis anos de idade, sendo 26 do sexo masculino e 24 do sexo feminino; 38 crianças com sete anos de idade, sendo 16 do sexo masculino e 22 do sexo feminino; e 42 crianças de oito anos de idade, sendo 24 do sexo masculino e 18 do sexo feminino. Uma análise por meio do teste de probabilidade exata de Fisher não evidenciou diferenças significativas quanto ao número de meninos e meninas que compuseram cada faixa etária investigada: 6 $\operatorname{anos}(p=0,5), 7$ anos $(p=0,32)$ e 8 anos $(p=0,33)$. O teste $\chi^{2}$ de uma variável foi utilizado para a verificação da adequação do número de participantes por faixa etária e não demonstrou diferenças significativas entre os mesmos: $\chi^{2}(\mathrm{df}=2)=1,74$; $p=0,42$.

\section{Materiais e instrumentos}

Os estímulos foram apresentados em um computador Celeron 2.5 GHZ, $512 \mathrm{Mb}$ de RAM, Drive de CD e HD de $40 \mathrm{~Gb}$, disponível no laboratório de informática da escola. O software E-Prime 2.0 (Schneider, Eschman \& Zuccolotto, 2002) foi utilizado na programação e na apresentação dos estímulos. O mesmo aplicativo registrou os tempos de reação (TRs) e os erros cometidos durante a tarefa. As respostas foram registradas com o auxílio de uma caixa de resposta serial (SRBOX), que é um hardware auxiliar do programa E-Prime 2.0. Os estímulos visuais foram compostos por 31 desenhos estilizados de objetos em preto e branco de dimensões equivalentes. 


\section{Procedimento}

O presente protocolo de pesquisa foi aprovado pelo Comitê de Ética em Pesquisa para seres humanos sob o número 126/11. A participação das 161 crianças foi previamente autorizado por meio do Termo de Consentimento Livre e Esclarecido (TCLC), devidamente firmado por seus responsáveis legais. O TCLC expunha os objetivos da pesquisa e o caráter livre da participação da criança, sem qualquer tipo de coação caso a criança desistisse de sua participação durante a realização das provas. As crianças também foram convidadas verbalmente a participar da pesquisa em linguagem condizente à idade.

A presente pesquisa delimitou duas variáveis independentes, idade e sexo. Três faixas etárias foram definidas (seis, sete e oito anos), resultando em um delineamento $3 \times 2$, analisado por meio do teste ANOVA para medidas repetidas. As variáveis dependentes analisadas foram: a velocidade de processamento (tempo de reação) e a taxa de erros (omissões ou coomissões). As omissões foram consideradas como a ausência de respostas nas tarefas agir, em que estímulos de objetos foram apresentados, enquanto que as coomissões consistiram nas respostas emitidas na apresentação do estímulo não agir (representado pela figura de uma bola).

As crianças foram convidadas individualmente a participar da tarefa e a sessão era iniciada com a apresentação de uma história, um conto, que contextualizava a tarefa da seguinte forma:

Sejam bem-vindos! Você está vendo este príncipe? (a pesquisadora apontava para o desenho de um príncipe estilizado na tela do computador). Ele quer sair para brincar com sua bola. Antes disso, no entanto, ele terá que organizar e guardar vários objetos que estão espalhados por todo o reino. É você quem vai ajudá-lo a organizar os objetos.
Você deverá apertar este botão (a pesquisadora apontava para a caixa de resposta), o mais rápido que puder, quando aparecerem os objetos para organizá-los! Só tem um detalhe: você não poderá apertar esse botão quando a bola aparecer, porque senão o príncipe não poderá brincar com ela depois se você a guardar! Então, quando aparecerem os objetos aqui na tela o que você vai fazer? (a pesquisadora aguardava a resposta das crianças e, se necessário, esclarecia dúvidas sobre a tarefa). Isso mesmo! E quando aparecer a bola, o que você deve fazer? (aguardava a resposta da criança). Isso mesmo! Então, vamos começar? Pode apertar o botão para começar!

Foram apresentadas 10 provas-treino seguidas do seguinte aviso: "Parabéns, você entendeu direitinho como ajudar o príncipe! Agora vamos começar?”. Caso a criança respondesse positivamente, a sessão era iniciada. Cada prova era iniciada com a apresentação de um ponto de fixação que permanecia na tela por $1000 \mathrm{~ms}$. Em seguida, o ponto de fixação era substituído pelo desenho de um objeto por 240 ms. Após a apresentação do objeto, uma máscara visual era apresentada até a resposta do participante ou por um intervalo de 2000 ms, conforme apresentado na Figura 1. Após o registro da resposta, era apresentada a mesma máscara na cor azul, caso a criança respondesse corretamente, e na cor vermelha, caso a criança respondesse incorretamente (feedback). A tarefa do participante era pressionar o botão central de uma caixa de resposta (agir), o mais rapidamente possível, cada vez que o desenho de um objeto fosse apresentado ( $81 \%$ das provas). Apenas com a apresentação do desenho de uma bola (19\% das provas), a resposta motora do participante deveria ser inibida (não agir). O experimento teve a duração média de $7 \mathrm{~min}$, nos quais os 30 estímulos agir foram apresentados cinco vezes, de forma aleatória, totalizando 150 apresentações. O estímulo não agir (bola) foi apresentado 35 vezes no total.

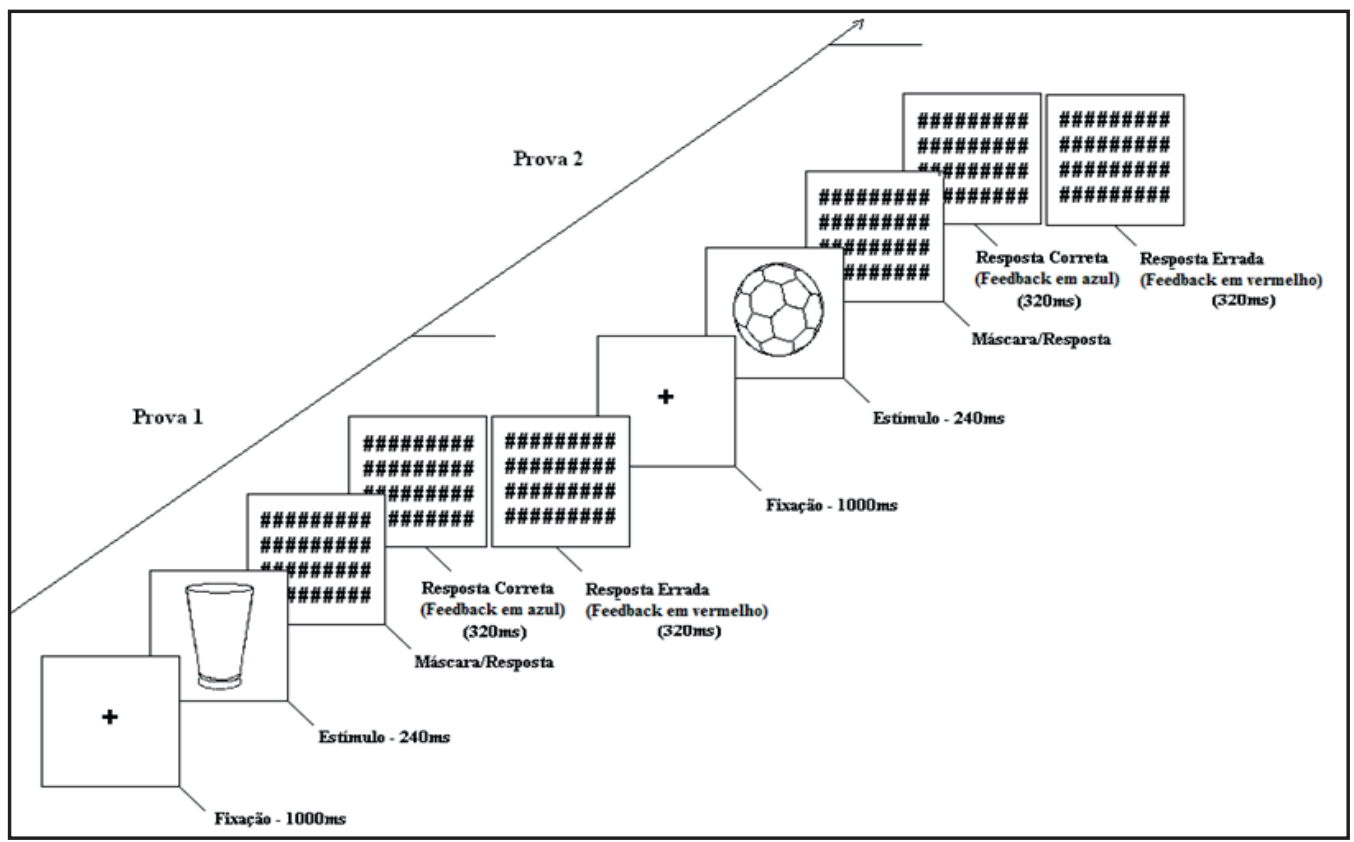

Figura 1. Representação esquemática da sequência de provas do Experimento $1(\mathrm{~ms}=$ tempo de duração da prova em milissegundos). 


\section{Resultados}

O tempo de reação (TR), a porcentagem de erros de omissão (respostas não efetuadas) e os erros de coomissão (respostas indevidamente efetuadas) foram submetidos à análise de variância para medidas repetidas (ANOVA $3 \times 2$ ). As provas em que o TR excedeu dois desvios padrão acima da média do tempo de resposta médio do participante, ou aquelas com TRs iguais ou inferiores a $100 \mathrm{~ms}$, foram excluídas da análise. A análise considerou dois fatores, idade (seis, sete e oito anos) e sexo (masculino e feminino), confirmando um efeito significativo no TR em função do fator idade $\left[\mathrm{F}(2,30)=4,08 ; \mathrm{p}=0,02 ; \eta^{2} \mathrm{p}=0,21 ; \mathrm{TR}\right.$ médio 6 anos $=585$ $\mathrm{ms}, \mathrm{SE}=47 \mathrm{~ms}$; TR médio 7 anos $=531 \mathrm{~ms}, \mathrm{SE}=30 \mathrm{~ms}$; TR médio 8 anos $=465 \mathrm{~ms}, \mathrm{SE}=30 \mathrm{~ms}$ ), como mostra a Figura 2a. O TR não variou significativamente em função do fator sexo $[F(1,15)=0,006 ; p=0,93]$ e não houve interação entre os fatores investigados $[\mathrm{F}(2,30)=2,17 ; \mathrm{p}=0,13]$. Os TRs em função do fator idade foram analisados por meio do teste post hoc de Newman-Keuls que evidenciou uma diferença significativa no desempenho entre crianças de 6 e 8 anos $(\mathrm{p}=0,02)$. Não houve diferença significativa nos TRs médios entre crianças de 6 e 7 anos $(p=0,3)$ e 7 e 8 anos $(p=0,08)$, como indicado na Figura 2.

Uma análise similar foi realizada com os erros de omissão (ANOVA 3×2). Essa análise evidenciou diferenças significativas no número de omissões em função do fator idade $\left[\mathrm{F}(2,30)=6,95 ; \mathrm{p}<0,01 ; \eta^{2} \mathrm{p}=0,31 ; 6\right.$ anos $=3,12 \% ; 7$ anos $=1,66 \% ; 8$ anos $=0,66 \%$ ], como mostra a Figura $2 b$. Não houve diferença significativa no número de omissões em função do fator sexo $[\mathrm{F}(1,15)=0,04 ; \mathrm{p}=0,82]$. Essa análise não evidenciou efeito de interação entre os fatores investigados $[F(2,30)=0,51 ; p=0,6]$. O teste post hoc de Newman-Keuls, realizado para o fator idade, confirmou uma diferença significativa no número de omissões entre crianças de seis e sete anos $(p=0,03)$ e entre crianças de seis e oito anos $(\mathrm{p}=0,002)$. Não houve diferença significativa no número de omissões entre crianças de sete e oito anos $(\mathrm{p}=0,14)$.

A mesma análise realizada com os erros de coomissão confirmou um efeito significativo em função do fator idade $\left[F(2,30)=11,45 ; p<0,001 ; \eta^{2} p=0,43 ; 6\right.$ anos $=16,5 \% ; 7$ anos $=11,3 \%$; 8 anos $=9,5 \%$ ), como mostra a Figura $2 b$. Não houve diferença significativa em função do fator sexo $[F(1,15)=1,79 ; p=0,2]$ e não houve interação entre os fatores investigados $[\mathrm{F}(2,30)=0,71 ; \mathrm{p}=0,5]$. A análise do fator idade pelo teste post hoc de Newman-Keuls confirmou uma diferença significativa entre crianças de 6 e 7 anos $(p=0,001)$ e entre crianças de seis e oito anos $(p<0,001)$. Não houve diferença significativa no número de coomissões entre crianças de sete e oito anos $(\mathrm{p}=0,3)$.

\section{Discussão}

De modo geral, os resultados observados no presente estudo contribuem para a compreensão do processo de desenvolvimento cognitivo do sistema atentivo e dos processos inibitórios envolvidos no controle da ação motora na infância. Foram observadas diferenças significativas na velocidade de processamento entre crianças de seis e oito anos de idade. A análise da frequência dos erros cometidos em função do fator idade também sugere que mudanças cognitivas importantes ocorrem nesta fase da infância. Diversos estudos apontam a ocorrência de um desenvolvimento acentuado na capacidade cognitiva na faixa etária compreendida entre cinco e oito anos de idade, permitindo uma melhora na capacidade adaptativa (social e emocional) das crianças nessa etapa do desenvolvimento (Bartgis et al., 2008).

De acordo com vários modelos de desenvolvimento da capacidade atentiva, a autorregulação e a articulação de

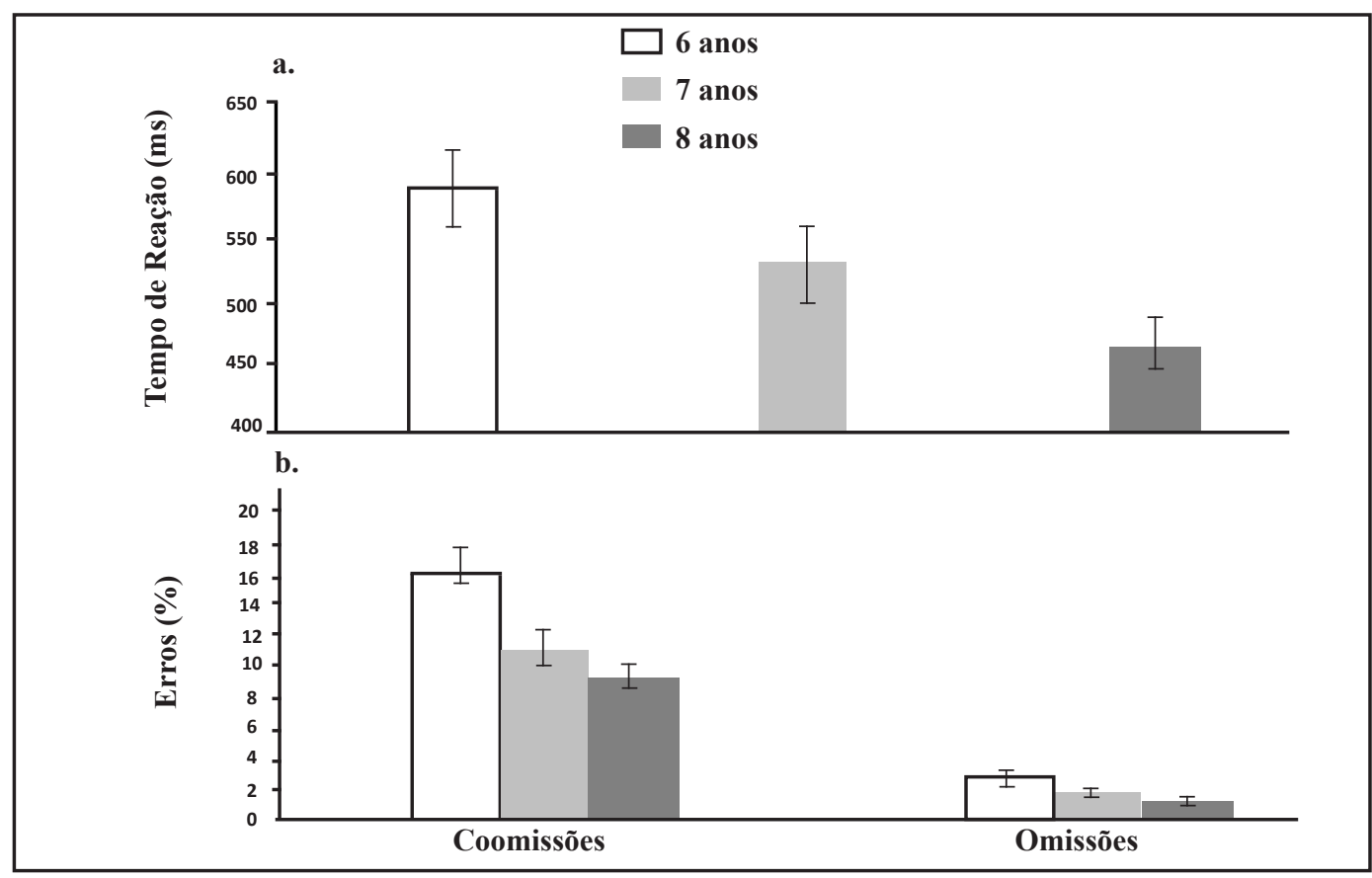

Figura 2. (a) Tempo de reação (ms) dos participantes em função do fator idade e (b) Porcentagem de erros de omissão e de coomissão efetuados em função do fator idade. 
diversos componentes executivos são fundamentais para a adaptação comportamental bem sucedida. Dentre esses compomentes, aspectos como a capacidade de inibição de uma resposta predominante, a capacidade de interrupção de uma resposta imediata em prol de um ganho a médio e a longo prazo, o controle de interferências (exógenas e endógenas) e a seleção adequada da informação, são fundamentais para o estabelecimento de padrões funcionais adequadamente adaptados (Barkley, 1997; Nigg, 2000). Vários estudos sugerem que esses aspectos melhoram significativamente com o aumento da idade (Bartgis et al., 2008; Davis et al., 2003; Miranda et al., 2009; Ridderinkhof \& van der Molen, 1995; van der Molen, 2000). Os resultados da presente investigação corroboram esses achados e sugerem uma melhora significativa desses processos com o aumento da idade na faixa etária investigada. De modo geral, as crianças de seis anos apresentaram, em média, respostas lentas quando comparadas às respostas efetuadas pelas crianças de oito anos.

Em relação à porcentagem de omissões cometidas, as crianças de seis anos cometeram significativamente mais omissões quando comparadas às crianças de sete e oito anos, o que corrobora os achados de outros trabalhos que evidenciam processos cognitivos atentivos e de controle inibitórios menos desenvolvidos na idade de 6 anos (Bartgis et al., 2003; Davis et al., 2003; Ridderinkhof \& van der Molen, 1995). Por outro lado, não foram observadas diferenças significativas entre o número de omissões cometidas pelas crianças de sete e oito anos, o que sugere, portanto, um desenvolvimento acentuado na capacidade de atenção seletiva (Epstein et al., 2003) entre as idades de seis e sete anos. No estudo de Rubia, Taylor, et al. (2001), crianças com TDAH também apresentaram maior taxa de erros relacionados às omissões em tarefa de inibição motora, incluindo a tarefa agir/não agir evidenciando a necessidade de comparação dos resultados desse estudo com amostras clínicas, além de discussões de possíveis diferenças significativas entre essas taxas de erros.

A análise da porcentagem de erros de coomissão também sugere um processo de maturação do controle inibitório ao longo da faixa etária investigada, ou seja, assim como na análise da porcentagem de omissões, as crianças de seis anos cometeram significativamente mais erros de coomissões quando comparadas às crianças de sete e oito anos. Não houve diferença na porcentagem de coomissões cometidas entre crianças de sete e oito anos, o que sugere uma melhora significativa do controle da ação entre as idades de seis e sete anos. Os resultados não evidenciaram diferenças significativas no desempenho em função do fator sexo.

Assim, os achados do presente estudo apontam que a tarefa agir/não agir delineada foi capaz de evidenciar aspectos distintos do desenvolvimento atentivo (atenção/desatenção e capacidade inibitória/impulvidade) ao longo de uma faixa etária bastante restrita do desenvolvimento (6-8 anos de idade). Nesse sentido, o estudo de Tsujimoto, Kuwajima e Sawaguchi (2007) aponta que as funções executivas, responsáveis pela inibição de respostas e pela memória de trabalho, apresenta um desenvolvimento fracionado durante a infância, o que permite a aquisição sucessiva de habilidades cognitivas cada vez mais complexas. Cragg e Nation (2008) também apresentam resultados que sustentam a interpretação que, na tarefa agir/não agir, as crianças mais novas (entre a faixa etária de cinco a sete anos) apresentam maior dificuldade em suprimir a resposta na presença de um estímulo não agir, comparadas às crianças na faixa etária de nove a 11 anos. De acordo com Bezdjian et al. (2009) e Epstein et al. (2003), as análises dos erros de omissão e coomissão na tarefa agir/não agir são significativamente correlacionados a comportamentos desatentos e comportamentos hiperativos/ impulsivos, respectivamente.

Em linhas gerais, os resultados da presente pesquisa corroboram os dados e os índices comportamentais de validação para o teste K-CPT (Conners, 2002) obtidos por Miranda et al. (2009) em uma amostra brasileira de crianças de 4 a 5 anos de idade. Todavia, os resultados não confirmaram diferenças significativas em função do fator sexo, como encontrado por Miranda et al. (2009). Uma possível razão para a não observação de diferenças significativas no desempenho em função do fator sexo, no presente estudo, pode estar relacionada às diferenças na faixa etária investigada nos dois estudos, uma vez que a amostra do estudo de Miranda et al. (2009) foi composta por crianças entre quatro e cinco anos, enquanto que a presente investigação foi realizada com uma amostra de crianças com idades entre seis e oito anos. A influência do fator sexo nesta etapa de maturação dos recursos cognitivos necessários para o controle inibitório efetivo ainda é uma questão controversa e aponta a necessidade de novos estudos que venham a ampliar a compreensão da influência desse fator nessa fase do desenvolvimento.

De maneira geral, a tarefa utilizada mostrou-se válida para diferenciar o desempenho das crianças de 6 a 8 anos de idade em relação a uma melhora progressiva da capacidade inibitória e da atenção seletiva. Contudo, futuros estudos são necessários para a ampliação das características demográficas da amostra. Tal ampliação e a inclusão de outras faixas etárias serão fundamentais para uma compreensão mais ampla do desenvolvimento do controle inibitório e dos processos atentivos de seleção da informação ao longo do desenvolvimento humano.

\section{Referências}

Barkley, R. A. (1997). Behavioral inhibition, sustained attention, and executive functions: constructing a unifying theory of ADHD. Psychological Bulletin, 121, 65-94.

Barkley, R. A., Anastopoulos, A. D., Robin, A. L., Lovett, B. J., Smith, B. H., Cunningham, C. E., Shapiro, C. J., .. Hathway, W. (2008). Transtorno de déficit de atenção/hiperatividade: manual para diagnostico e tratamento ( $3^{\circ}$ ed.). Porto Alegre: Artmed

Bartgis, J., Lilly, A., \& Thomas, D. C. (2003). Event-related potential and behavioral measures of attention of 5, 7, and 9 years-old. Journal of General Psychology, 130(3), 311-335.

Bartgis, J., Thomas, D. G., Lefler, E. K., \& Hartung, C. M. (2008). The development of attention and response inhibition in early childhood. Infant and Child Development, 17, 491-502. 
Bezdjian, S., Baker, L. A., Lozano, D. I., \& Raine, A. (2009). Assessing inattention and impulsivity in children during the Go/NoGo task. British Journal Developmental Psychology, $27(2), 365-383$.

Conners, C. K. (2002). Conners' continuous performance test for Windows [Computer software]. Toronto: Multi-Health.

Cragg, L., \& Nation, K. (2008). Go or no-go? Developmental improvements in the efficiency of response inhibition in midchildhood. Developmental Science, 11(6), 819-827.

Davis, E. P., Bruce, J., Snyder, K., \& Nelson, C. A. (2003). The X-trials: Neural correlates of inhibitory control task in children and adults. Journal of Cognitive Neuroscience, 15, 432-443.

Epstein, J. T., Erkanli, A., Conners, C. K., Klaric, J., Costello, J. E., \& Angold, A. (2003). Relations between continuous performance test performance measures and ADHD behaviors. Journal of Abnormal Child Psychology, 31(5), 543-554.

Hamdan, A. C., \& Pereira, A. P. A. (2009). Avaliação neuropsicológica das funções executivas: considerações metodológicas. Psicologia: Reflexão e Crítica, 22(3), 386-393.

Jaeger, A. (2013). Inhibitory control and the adolescent brain: a review of MRI research. Psychology \& Neuroscience, 6(1), 23-30.

Johnstone, S. J., Pleffer, C. B., Barry, R. J., Clarke, A. R., \& Smith, J. L. (2005). Development of inhibitory processing during the Go/NoGo Task: A behavioral and event-related potential study of children and adults. Journal of Psychophysiology, 19(1), 11-23.

Klein, R. M. (2000). Inhibition of return. Trends in Cognitive Sciences, 4(4), 138-146.

Korkman, M., Kemp, S. L., \& Kirk, U. (2001). Effects of age on neurocognitive measures of children ages 5 to 12 : A crosssectional study on 800 children from the United States. Developmental Neuropsychology, 20(1), 331-354.

Logan, G. D., \& Cowan, W. B. (1984). On the ability to inhibit thought and action: A theory of an act of control. Psychological Review, 91(3), 295-327.

Logan, G. D. (1994). On the ability to inhibit thought and action -A user's guide to the stop signal paradigm. In D. Dagenbach, \& T. H. Carr (Eds.), Inhibitory processes in attention, memory, and language (pp. 189-239). San Diego, CA: Academic Press.

Miranda, M. C., Sinnes, E. G., Pompeia, S., \& Bueno, O. F. A. (2009). O K-CPT em uma amostra brasileira: Descrição do desempenho e comparação com as normas norte-americanas. Revista de Psiquiatria do Rio Grande do Sul, 31(1), 60-66.
Newman, J. P., Widom, C. S., \& Nathan, S. (1985). Passive avoidance in syndromes of disinhibition: Psychopathy and extraversion. Journal of Personality and Social Psychology, 48(5), 1316-1327.

Nigg, J. T. (2000). On inhibition/disinhibition in developmental psychopathology: Views from cognitive and personality psychology and a working inhibition taxonomy. Psychological Bulletin, 126(2), 220-246.

Riccio, C. A. Reynolds, C. R., \& Lowe, P. A. (2001). Clinical applications of continuous performance tests: Measuring attention and impulsive responding in children and adults. New York, NY: John Wiley \& Sons, Inc.

Ridderinkhof, K. R., \& van der Molen, M. W. (1995). A psychophysiological analysis of developmental differences in the ability to resist interference. Child Development, 66(4), 1040-1056.

Roberts, R. J., \& Pennington, B. F. (1996). An interactive framework for examining prefrontal cognitive processes. Developmental Neuropsychology, 12(1), 105-126.

Rubia, K., Russell, T., Overmeyer, S., Brammer, M. J., Bullmore, E. T., Sharma, T., . . \& \& Taylor, E. (2001). Mapping Motor Inhibition: Conjunctive brain activations across different versions of Go/No-Go and Stop Tasks. NeuroImage, 13, 250-261.

Rubia, K., Taylor, E., Smith, A. B., Oksannen, H., Overmeyer, S., \& Newman S. (2001). Neuropsychological analyses of impulsiveness in childhood hyperactivity. The British Journal of Psychiatry, 179, 138-143.

Rubia, K., Smith, A. B., Woolley, J., Nosarti, C., Heyman, I., Taylor, E., \& Brammer, M. J. (2006). Progressive increase of frontostriatal brain activation from childhood to adulthood during event-related tasks of cognitive control. Human Brain Mapping, 27, 973-993.

Schneider, W., Eschman, A., \& Zuccolotto, A. (2002). E-Prime (Version 2.0) [Computer software and manual]. Pittsburgh, PA: Psychology Software Tools Inc.

Tsujimoto, S., Kuwajima, M., \& Sawaguchi, T. (2007). Developmental fractionation of working memory and response inhibition during childhood. Experimental Psychology, 54(1), 30-37.

Van der Molen, M. W. (2000). Developmental changes in inhibitory processing: Evidence from psychophysiological measures. Biological Psychology, 54, 207-239.

Verbruggen, F., \& Logan, G. D. (2008). Automatic and controlled response inhibition: Associative learning in the Go/No-Go and Stop-Signal Paradigms. Journal of Experimental Psychology: General, 137(4), 649-672. 